\title{
El derecho cosmopolita de Fichte a la luz de la crítica de Hegel al Grundlage des Naturrechts
}

\section{Héctor Oscar Arrese lgor}

CONICET, Argentina

\begin{abstract}
Resumen
Hegel desarrolló hacia 1802/1803 una crítica radical del derecho natural del Fichte de Jena. En especial intentó mostrar que el supuesto del egoísmo universal, sobre el cual Fichte construye su sistema del derecho aplicado, no puede sostener el funcionamiento de la comunidad política, porque es necesario apelar a instancias externas, tales como la virtud de los éforos o de los éforos naturales. En este trabajo intento mostrar que el argumento de Hegel también podría aplicarse al ámbito del derecho internacional fichteano. La razón de esto es que allí también Fichte opera con el supuesto del egoísmo universal, a fin de construir un sistema que disuada a los Estados de atacar a los demás, por miedo a la coacción representada por la declaración de guerra. Pero la amenaza constante del ataque de los demás Estados, producto del supuesto del egoísmo universal, impide la construcción de un orden mundial pacífico donde se respeten los derechos de todos.
\end{abstract}

\begin{abstract}
In 1802-1803, Hegel developed a radical criticism of Fichte's natural right of Jena, in which he explained that the assumption of universal egoism, upon which Fichte founds his entire system of applied right, cannot support the functioning of the political community. On the contrary, Hegel argued, it is necessary to draw upon externalities such as the virtues of either the ephors or natural ephors. In this paper, I show that Hegel's argument is also applicable to Fichte's international right, for regarding that realm Fichte also assumes universal egoism, chiefly in order to construct a system that discourages states from attacking others out of fear of coercion represented in a possible declaration of war. Yet, the constant threat of such attacks prevents society from cultivating a peaceful world order-one in which the rights of all are respected.
\end{abstract}

Palabras clave

Hegel Fichte estado derecho cosmopolitismo
Keywords

Hegel Fichte state right cosmopolitism
El tratado de Johann Gottlieb Fichte, titulado como Grundlage des Naturrechts nach Principien der Wissenschaftslehre, generó debates y controversias ya desde su publicación, hacia 1796 y 1797. En especial, Georg Wilhelm Friedrich Hegel puso en cuestión la teoría fichteana del Estado en su famoso Überdie wissenschaftlichen Behandlungsarten des Naturrechts, seine Stelle in der praktischen Philosophie und sein Verhältnis zu den positiven 
Rechtswissenschaften (Hegel, 1802/1803). El texto, que vio la luz entre 1802 y 1803 , apunta sobre todo a la concepción del derecho de coacción, que es el eje estructurante de la idea del Estado. La tesis central de Hegel consiste en que la teoría fichteana no logra su cometido fundamental, que es la garantía de los derechos como una forma de reconocimiento del otro.

Como veremos más adelante, Fichte intenta construir una idea del Estado que haga posible que los ciudadanos delimiten mutuamente sus esferas de acción, a fin de transmitirse los unos a los otros la concepción que cada uno tiene del otro como un ser racional y libre. Pero, a la hora de diseñar un sistema de derechos que haga posible este ideal, Fichte presupone que los ciudadanos son o pueden llegar a ser egoístas racionales, por lo que se ve obligado a suponer que respetarán la ley únicamente para proteger su libertad y su propiedad. Esta idea del derecho de coacción se constituye en un sistema que funciona con una precisión casi mecánica. La crítica de Hegel apunta a mostrar que el Estado fichteano se convierte en una máquina en vez de un organismo vivo, en el que cada ciudadano pueda integrarse como una parte del todo. En este trabajo reconstruiré en primer lugar la crítica de Hegel al derecho de coacción. Finalmente, defenderé la hipótesis de que los problemas que Hegel señala en el ámbito de la teoría del Estado también tienen lugar en el derecho internacional, del cual Hegel no se ocupa en su texto.

\section{La crítica de Hegel al derecho fichteano de coacción}

A fin de estar en condiciones de poner la crítica de Hegel a prueba en el ámbito del derecho internacional, es necesario ante todo reconstruir la argumentación pensada originalmente para el ámbito del derecho intraestatal. A su vez, no podremos comprender las objeciones hegelianas si antes no consideramos los momentos fundamentales de la teoría de Fichte.

El objetivo central del derecho natural fichteano consiste en garantizar las condiciones fundamentales para la realización de la autoconciencia, entendida como la atribución del yo a sí mismo de una determinada eficacia en el mundo sensible (Fichte, 1796/1797: 17). A partir de un estudio minucioso de este problema desde el parágrafo primero al tercero del Grundlage des Naturrechts, Fichte concluye que la autoconciencia no es posible si el yo no es reconocido por otro individuo como un ser racional y libre, dado que la autoconciencia no contiene en sí misma las condiciones para su puesta en marcha (Fichte, 1796/1797: 34).

El yo puede saber que es un sujeto capaz de actuar en el mundo sensible sólo si otro le transmite este conocimiento por medio de una relación intersubjetiva particular, que consiste en la exhortación (Aufforderung) del otro al yo para que se determine a sí mismo a actuar en el mundo sensible. Se trata de una relación intersubjetiva diferente de la objetual, porque el otro no restringe la libertad del yo, sino que la potencia, dado que apunta a que el yo descubra sus capacidades en tanto que ser racional y libre.

Ahora bien, para que efectivamente se trate de una relación de reconocimiento mutuo, el otro no debe ejercer ningún tipo de fuerza sobre el yo, quien debe ser libre de responder o no a la exhortación del otro. Esto significa que el otro debe dejar abierta al yo una esfera de acciones libres, dado que el reconocimiento del otro como un sujeto libre es contradictorio con la coacción para que actúe de tal o cual manera (Fichte, 1796/1797: 34). En consecuencia, esta relación intersubjetiva debe estar regida entonces por el principio del derecho (Rechtssatz), que prescribe a cada sujeto la limitación de una esfera de acciones libres para sí mismo que debe ser igual a aquella que él respeta en los demás (Fichte, 1796/1797: 52). 
Ahora bien, esta relación de mutuo reconocimiento de derechos contiene en sí una cierta indeterminación, porque presupone que los sujetos son libres en todo tiempo de seguir reconociendo al otro como un sujeto de derechos o de dejar de hacerlo. En caso contrario, estaríamos frente a una relación de coacción y no de exhortación y reconocimiento del otro como un sujeto libre. Pero esta indeterminación pone en peligro a su vez la estabilidad de la comunidad legal a futuro.

La relación legal debe ser estable y permanente porque cada yo debe ponerse a sí mismo como un agente para todo el tiempo futuro, dado que en caso contrario caería en contradicción consigo mismo, toda vez que no puede ponerse como un sujeto en un momento determinado y negarse a sí mismo este status en un momento posterior (Fichte, 1796/1797: 58). Por lo tanto, el yo debe tener la garantía de que será reconocido por los demás como un sujeto de derecho para todo tiempo futuro. Es decir que, si los demás no lo reconocieran, caerían también en contradicción consigo mismos, dado que no pueden afirmar que un sujeto es libre en un momento y negarlo acto seguido.

Por lo tanto, la racionalidad misma, es decir la necesidad de coherencia entre las acciones (lo que Fichte denomina como la Einstimmigkeit mit sich selbst) exige que la comunidad legal establecida por los sujetos tenga una permanencia y estabilidad a futuro. Como consecuencia, surge otro problema, dado que es necesario establecer la instancia que garantizará los derechos de los sujetos. Ésta no puede estar constituida por ninguno de los participantes de la comunidad legal, porque en caso contrario no podrían garantizar la imparcialidad a la hora de dirimir los conflictos de derechos, dado que serían una parte interesada en ellos. Debe tratase por lo tanto de una tercera persona, que no tenga interés en defender a ninguna de las partes en particular (Fichte, 1796/1797: 157). En suma, debe entrar en juego la instancia de lo público, representada en la persona del gobernante.

El Estado debe garantizar entonces el derecho a una esfera de acciones libres, que exige ante todo el derecho a la autoconservación en la existencia (lo que Fichte denomina como un Existenzminimum) (Fichte, 1796/1797: 212). Fichte fundamenta la necesidad de un derecho a la existencia en la estructura misma de la acción. Cuando el yo se propone realizar una acción, debe necesariamente prever que él mismo al menos existirá al momento de lograr su objetivo. Por lo tanto, el derecho a la actividad libre implica necesariamente el derecho a la autoconservación.

El Estado entra en escena para garantizar el derecho en la eventualidad de que alguna de las partes desistiera de cumplir el compromiso que la llevó a entrar en la relación legal. Por lo tanto, el gobernante debe actuar como si todos los ciudadanos fueran egoístas, para minimizar el riesgo de que ocurra dicha eventualidad. Esto exige un control férreo de la distribución del ingreso y una planificación centralizada de la división del trabajo.

El sistema legal ideado por Fichte parte de este supuesto del egoísmo universal, y funciona de modo tal que utiliza el autointerés de los ciudadanos para estimularlos a cumplir con la ley por miedo al perjuicio que, en caso contrario, les ocasionarían las sanciones a su libertad y propiedad (Fichte, 1796/1797: 141). ${ }^{1}$ Esta concepción del castigo es denominada por Fichte como derecho de coacción (Zwangsrecht).

La crítica de Hegel se dirige al derecho de coacción mismo, en tanto que tiene la función de garantizar los derechos necesarios para que la relación de mutuo reconocimiento tenga lugar. Hegel objeta que la libertad no consiste en la mera protección de los derechos de la libertad de acción y la propiedad, tal como Fichte argumenta. Por el contrario, la idea hegeliana consiste en que la libertad tiene lugar cuando el individuo se siente parte de la comunidad política (Hegel, 1802/1803: 477). Para que
1. En este punto ejerció una influencia importante la obra Apologie des Teufels, publicada en 1795 por Johann Benjamin Erhard; Oncina Coves (1994: 329); Erhard (1795), así como la lectura de Machiavelli, ver Philonenko (2003: 53). Ver Fichte (1807: 420). 
esto ocurra, es fundamental que el sistema legal esté integrado con las tradiciones culturales, religiosas, etc., de la comunidad de pertenencia (Hegel, 1802/1803: 507). Es entonces el espíritu del pueblo quien debe estructurar las normas legales que rigen la convivencia, para que puedan anclar en las convicciones morales compartidas por todos los ciudadanos (Hegel, 1802/1803: 503).

Por estas razones es que Hegel impugna el derecho de coacción fichteano, en la medida en que se funda en el supuesto del egoísmo universal. El supuesto del egoísmo presupone que ya se ha roto la confianza mutua entre los ciudadanos y que, por lo tanto, la comunidad legal ya no es posible. Lo que queda, siguiendo la argumentación de Hegel, es una máquina que funciona en base a la coacción sobre los ciudadanos, es decir a su falta de libertad. Esto implica una concepción abstracta de la personalidad de los ciudadanos, dado que contempla únicamente la motivación basada en el auto-interés. En consecuencia, la teoría fichteana pierde de vista a los individuos de carne y hueso y, con ello, la posibilidad misma de ser aplicada a la vida real de los ciudadanos cuya libertad quiere garantizar. Por otro lado, la justicia se convierte en una mera contabilidad de delitos y castigos, esto es en una forma de venganza. Por lo tanto, la teoría termina alejándose de aquella comunidad inicial de respeto mutuo basada en la mutua exhortación a la acción.

Considero que la crítica de Hegel es pertinente, aún si no compartimos su concepción de la libertad, la moralidad y el derecho. Hegel muestra que el Estado fichteano, construido sobre el supuesto del egoísmo universal, no logra su objetivo primordial, porque se trata de una abstracción de los móviles reales de los ciudadanos. Esta debilidad de la teoría se hace evidente si tomamos en cuenta el problema del control de los actos de gobierno por parte del pueblo.

Fichte encara esta cuestión diseñando una forma de gobierno en la que los tres poderes estén centralizados en el ejecutivo, para que la aplicación de la ley pueda ser efectiva (Fichte, 1796/1797: 161). ${ }^{2}$ Los ciudadanos se retiran entonces a su vida cotidiana y se abstienen de participar en la vida política. Ahora bien, esto deja planteada la cuestión de cómo fiscalizar los actos de gobierno e intervenir en el caso de que el gobernante traicione el mandato popular de respetar la libertad y la propiedad de todos. Con esta finalidad, Fichte introduce una institución de hombres de probada prudencia y sabiduría, a la que denomina el eforato (Fichte, 1796/1797: 170-173). Este cuerpo tiene la obligación de dictar un interdicto en caso de injusticia flagrante y llamar a la asamblea popular para celebrar el juicio político contra el gobierno (Fichte, 1796/1797: 171).

Pero, a pesar de este mecanismo, no habría garantías de que el eforato no sea corrompido, amenazado o extorsionado por el gobierno. En este caso, la teoría no dispone de un reaseguro, dado que el sistema descansa sobre el egoísmo racional de los ciudadanos y la virtud de los éforos. Si los ciudadanos controlaran al gobierno por medio de personas tan egoístas como ellos, entonces estas personas tratarían de obtener ventajas de su lugar de poder frente al gobierno, por lo que rápidamente se corrompería y no cumpliría con sus funciones. Al no hacerlo, se caería el sistema mismo, que descansa sobre la protección de los derechos de los ciudadanos por parte del gobierno.

En esta situación límite Fichte se ve obligado a recurrir a algunos ciudadanos virtuosos, a quienes denomina como los "éforos naturales". En caso de que la sociedad no esté también totalmente corrupta, Fichte cuenta con esta reserva moral de ciudadanos que se alzarán contra el eforato y el gobierno, denunciando su traición a los principios de la Constitución (Fichte, 1796/1797: 182-183). Es decir que, tal como Hegel argumenta, el supuesto del egoísmo universal, que está llamado a sostener el sistema de Fichte, no puede hacerlo, en razón de su carácter abstracto. Por lo tanto, Fichte debe fundar el sistema legal en instancias externas al mismo, como son los hombres virtuosos que integran el eforato o los éforos naturales. 


\section{La crítica de Hegel a la luz del derecho internacional fichteano}

La teoría del derecho internacional fue pensada por Fichte como un apéndice al derecho natural. Sin embargo, es un momento de la obra que permite poner a prueba algunas tesis fundamentales de su teoría pura del derecho y del Estado. Aquí intentaré mostrar que la crítica de Hegel al derecho de coacción también podría extenderse al derecho internacional, que no es tomado en cuenta en la serie de objeciones esgrimidas.

El punto de partida de la argumentación de Fichte consiste en la constatación de que el Estado no puede bastarse a sí mismo, porque los ciudadanos necesariamente entrarán también en contacto con ciudadanos de otros Estados. Cuando ocurra esto, resultará evidente que cada ciudadano tendrá derecho a coaccionar a un extranjero que entre en relaciones con él para que se someta a su propio Estado. La razón de esto es que sólo su propio Estado puede garantizarle la libertad y la propiedad y, en razón del principio del derecho, no está obligado a ceder su esfera de acciones libres a nadie (Fichte, 1796/1797: 369). ${ }^{3}$ Sin embargo, esta exigencia sería contradictoria en una tal situación, porque cada ciudadano tendría un legítimo derecho a coaccionar al otro para que se integre a su Estado, con lo que ambas exigencias se anularían mutuamente.

La única solución posible a este problema, desde el punto de vista de Fichte, consiste en que, en el caso de que un ciudadano atentara contra el derecho de otro ciudadano extranjero, sea castigado por el juez de su propio Estado, exactamente del mismo modo en que si hubiera agredido a un compatriota (Fichte, 1796/1797: 370-371). Pero esta protección legal a los extranjeros puede ser garantizada únicamente bajo la forma de un contrato formulado explícitamente entre los Estados. La cláusula fundamental de este contrato es: “(...) me hago responsable de todos los daños que mis conciudadanos pudieran acarrear a los tuyos, bajo la condición de que seas igualmente responsable de todos los daños que tus ciudadanos pudieran acarrear a los míos" (Fichte, 1995: 413). ${ }^{4}$

Este contrato no funciona del mismo modo que el contrato de ciudadanía, porque los Estados no pueden ser integrados en un Estado mayor, dado que perderían su autonomía y no podrían cumplir con la función que le ha sido delegada por los ciudadanos, esto es la protección de su libertad y su propiedad. La reciprocidad propia de este contrato interestatal implica que cada Estado se ve obligado a proteger los derechos de los demás ciudadanos sólo si los demás hacen lo propio. ${ }^{5}$ Esto implica que cada Estado, a la hora de juzgar los delitos contra ciudadanos extranjeros, deberá evaluar si el otro Estado involucrado también está dispuesto a proteger los derechos de los ciudadanos del primero. ${ }^{6}$ Para ello, ante todo deberá considerar si al menos el Estado puede respetar el derecho en sus relaciones con los demás, para lo que debe tener una cierta legalidad (Fichte, 1796/1797: 372). Por otro lado, si un Estado no reconoce la legalidad de otro, entonces está legitimado para declararle la guerra (Fichte, 1796/1797: 372-373). ${ }^{7}$

El Estado que no respeta el derecho de los demás puede ser sojuzgado sin límite alguno, es decir que pierde todos sus derechos, porque no es confiable frente a los otros Estados (Fichte, 1995: 418)..$^{8}$ La guerra entonces toma la forma del derecho de coacción, que utiliza el egoísmo de los gobernantes para que no atenten contra la libertad de los demás Estados, por temor a perder su autodeterminación. ${ }^{9}$

Para que la guerra sea un medio disuasorio efectivo, los Estados que firman el contrato interestatal deben sumar sus fuerzas armadas respectivas para constituir una fuerza que sea superior a la de cada uno en particular (Fichte, 1796/1797: 379). ${ }^{10}$ Esta fuerza no puede estar a cargo de un Estado integrado por todos los Estados particulares (Völkerstaat), porque esto implicaría la cesión de la soberanía de estas comunidades, sino más bien una federación de pueblos (Völkerbund). Es decir que se trata
3. Fichte parte de los conflictos que se dan entre los ciudadanos de diferentes Estados y de ahi plantea los problemas del derecho de gentes, dado que el Estado es un concepto abstracto y sólo los individuos son concretos y reales De Pascale (2001: 198).

4. "(...) ich mache mich verantwortlich für allen Schaden, den meine Bürger den deinigen zufügen könnten, unte der Bedingung, dass du gleichfalls verantwortlich seyst für allen Schaden, den deine Bürger den meinigen zufügen könnten" (Fichte, 1796/1797: 371).

5. Villacañas objeta con agudeza que la fundamentación de las relaciones pacíficas de los Estados en el cumplimiento de pactos de reciprocidad que no toman en cuenta otros factores también importantes a la hora de desencadenar conflictos internacionales. Puede hacerse referencia, por ejemplo, a mecanismos de integración interestatal de orden cultural, etc. Es decir que Fichte parte de la idea de Estados cerrados y autárquicos, concepción que luego se profundizará en Der geschlossene Handelstaat y que, por otro lado, está totalmente ausente del cosmopolitismo kantiano (Villacañas, 1999: 78, 101). 6. Como señala Von Manz, en el derecho cosmopolita del Grundlage des Naturrechts el objetivo es proteger a los ciudadanos cuando se les priva de sus derechos en otro Estado, pero no cuidar de que se respeten los derechos de los ciudadanos al interior de su propio Estado, lo que constituye el fin de cada Estado en sí (Von Manz, 2008: 191-192).

7. David James señala que la concepción fichteana del cosmopolitismo del derecho natural está más bien centrada en la garantía de la independencia de los Estados respecto de los demás, pero todavía no considera las asimetrías económicas que pueden llegar a darse en las relaciones internacionales. Fichte habría enfrentado esta problemática recién en Der geschlossene Handelstaat James (2010: 65-66).

8. "Also der natürliche Zweck des Krieges ist immer die Vernichtung des bekriegten Staats, d.i. die Unterwerfung seiner Bürger" (Fichte, 1796/1797: 377). Fichte sostiene que no puede probarse que la federación de Estados va a ser una organización justa pero que, sin embargo, es menos injusta que la guerra. Kleingeld señala acertadamente que esta afirmación muestra que Fichte no considera que esté justificada la creencia en que la federación de Estados traerá la paz perpetua (Kleingeld, 1999: 512).

9. Fichte utiliza entonces la misma lógica para el derecho estatal y para el internacional, buscando evitar la salida hobbesiana de la lucha de todos contra todos (Villacañas, 1996: 111).

10. Ver Fichte (1807: 425-426). 
11. Ver De Pascale (2001: 201). Como afirma Oncina Coves, dado que en Fichte la historia no tiene ningún mecanismo providencial que conduzca a la humanidad hacia la paz perpetua, es la libre decisión de los hombres y de los pueblos la que debe motivar la búsqueda de mecanismos en esta dirección (Oncina Coves, 1996: 169).

12. Como señala Philonenko, Fichte considera en su último período que las relaciones internacionales son relaciones de guerra, dado que todos los Estados buscan la hegemonía. Por lo tanto, ningún Estado puede confiar en la palabra de ningún otro (Philonenko, 2003: 55). Ver Fichte (1812: 648-649). de una unión en la que los Estados puedan ingresar voluntariamente, dado que también pueden mantenerse relaciones interestatales legítimas por fuera de esta organización (Fichte, 1796/1797: 379-380). ${ }^{11}$

El status legal de la federación de Estados no está en condiciones de eliminar las asimetrías de poder que puedan darse en el nivel internacional, porque se trata de una institución que no posee una instancia centralizada que pueda controlar los excesos de poder dentro de la misma. La federación de Estados opera con la dinámica del derecho de coacción, que Fichte postula para el derecho estatal, el que supone una autoridad que concentre el poder de decisión, dado que el egoísmo universal degeneraría en una lucha de todos contra todos.

Por el contrario, la federación de Estados consta de un Tribunal que fiscaliza la corrección de las cláusulas del contrato interestatal, el cumplimiento de las mismas y la eventual declaración y conducción de una guerra. Pero no existe un poder ejecutivo que limite el poder de extorsión que tendría, por ejemplo, un Estado con un armamento mucho más poderoso que los demás integrantes de la federación (Fichte, 1812: 645-646). De este modo, la guerra no puede aplicarse como un derecho de coacción en el marco de esta organización interestatal. ${ }^{12}$

Por lo tanto, tampoco puede cumplirse el principio del derecho, que apunta a garantizar una esfera igual de acción libre para cada sujeto. De allí que la relación simétrica de mutuo reconocimiento, esencial al derecho puro, no encuentra su expresión en el derecho internacional, en razón del supuesto del egoísmo universal.

Como consecuencia, el derecho no queda garantizado en el ámbito internacional y queda restringido al interior de cada Estado. Sin embargo, esto conlleva una consecuencia de peso mayor, porque si el derecho no tiene vigencia en las relaciones con los demás Estados, entonces el Estado mismo tampoco podrá hacerlo cumplir al interior de su territorio, dado que necesita de la cooperación de los demás gobiernos para disponer de la autodeterminación suficiente para poder cumplir con sus funciones.

De lo argumentado hasta aquí puede concluirse que el supuesto del egoísmo universal lleva tanto al colapso del derecho de coacción como del derecho internacional. Tal como lo ha señalado Hegel para el derecho de coacción, este supuesto psicológico-moral crea una situación extrema de incertidumbre y mutua desconfianza que hace necesario el control permanente de los ciudadanos. A su vez, esta situación impide su participación en la toma de decisiones relevantes para la comunidad, a causa del riesgo de fragmentación social que esto involucraría. Por esta razón, los ciudadanos pierden su libertad y quedan a merced de un gobierno que centraliza todo el poder. En consecuencia, queda latente la posibilidad de que el gobierno se corrompa e intente sobornar o amedrentar al eforato, que es la única instancia de control que le queda al pueblo. En el caso de que el eforato fracase, sólo podrá apelarse a la virtud y el sentido moral de un grupo de ciudadanos, esto es, los éforos naturales. Por lo tanto, el sistema basado en el egoísmo universal no puede sostenerse por sí mismo.

Algo análogo ocurre en el ámbito del derecho internacional, según lo expuesto más arriba. Por un lado, Fichte diseña un sistema de mutua protección entre los Estados para evitar que alguno de ellos viole los derechos de los ciudadanos de otro o avance en contra de la autodeterminación de otro Estado, por medio de invasiones o diferentes modos de agresión. Ahora bien, el supuesto del egoísmo universal impide establecer algún tipo de alianza duradera entre los Estados, porque siempre acechará la desconfianza de que el otro atacará en la primera oportunidad que se le presente. 
Por eso la guerra, que debería tener un efecto disuasorio sobre los posibles atacantes, puede convertirse en algo más usual que lo deseado, poniendo en jaque el sistema entero diseñado por Fichte para la convivencia entre los Estados.

De este modo, puede concluirse que la crítica de Hegel al derecho natural de Fichte es un aporte que puede ser aplicado de modo fructífero al derecho internacional, ampliando el alcance de la objeción original a un campo que será objeto de trabajo intensivo por parte de Fichte, una vez que haya abandonado Jena. 


\section{Q Bibliografía}

"De Pascale, C., (2001). Das Völkerrecht (Zweiter Anhang). En Merle, J. Ch., (Ed.). Johann Gottlieb Fichte. Grundlage des Naturrechts (197-210). Berlín: Akademie Verlag.

»Erhard, J. B., (1795). Apologie des Teufels, reimpreso en: Johann Benjamin Erhard. Über das Recht des Volks zu einer Revolution und andere Schriften (109-134), (1976). Frankfurt: Syndikat.

» Fichte, J.G., (1796/1797). Grundlage des Naturrechts nach der Principien der Wissenschaftslehre. Se sigue la paginación de la edición de Fichte, I. H., (ed.), (1845, ss.), Johann Gottlieb Fichte: Sämmtliche Werke. Editado por. Immanuel Hermann Fichte. Leipzig. Reeditado en: (1971). Berlín: Walter de Gruyter, Tomo III.

» Fichte, J. G., (1807). Ueber Macchiavelli, als Schriftsteller, und Stellen aus seinen Schriften. Se sigue la paginación de la edición de Fichte, I. H., (ed.), (1845, ss.), Johann Gottlieb Fichte: Sämmtliche Werke. Editado por Immanuel Hermann Fichte. Leipzig. Reeditado en: (1971). Berlín: Walter de Gruyter, Tomo X.

»Fichte, J. G., (1812). Das System der Rechtslehre. Se sigue la paginación de la edición de Fichte, I. H., (Ed.), (1845, ss.), Johann Gottlieb Fichte: Sämmtliche Werke. Editado por. Immanuel Hermann Fichte. Leipzig. Reeditado en: (1971). Berlín: Walter de Gruyter, Tomo X.

» Fichte, J. G., (1995). Fundamento del derecho natural según los principios de la dotrinca de la ciencia (Traducción española de José L. Villacañas Berlanga, Manuel Ramos Valera y Faustino Oncina Coves). Madrid: Centro de Estudios Constitucionales.

» Hegel, G. W. F., (1802)/(1803). Über die wissenschaftlichen Behandlungsarten des Naturrechts, seine Stelle in der praktischen Philosophie und sein Verhältnis zu den positiven Rechtswissenschaften; en: Kritisches Journal der Philosophie, Bd. II, Stück 2, [November/Dezember] 1802, und Stück 3, [Mai/Juni] 1803. Citado según la paginación de la edición: G.W.F. Hegel (1970). Werke in 20 Bänden. Band 2. Suhrkamp Verlag.

» James, D., (2010). Fichte's reappraisal of Kant's theory of cosmopolitan right. History of European Ideas, 36, 61-70.

" Kleingeld, P., (1999), Six Varieties of Cosmopolitanism in Late Eighteenth-Century Germany. Journal of the History of Ideas, Vol. 60, №3, 505-524.

"Oncina Coves, F., (1994). Para la paz perpetua de Kant y el Fundamento del derecho natural de Fichte: encuentros y desencuentros. Daimon. Revista de Filosofía, № 9 , 323-339.

» Oncina Coves, F., (1996). De la candidez de la paloma a la astucia de la serpiente; la recepción de la Paz Perpetua entre sus coetáneos. En Aramayo, R., Muguerza, J., Roldán, C., (Eds.). La paz y el ideal cosmopolita de la Ilustración. A propósito del bicentenario de Hacia la paz perpetua de Kant (155-189). Madrid: Tecnos.

» Oncina Coves, F., (2004). Fichtes Kritik des aufklärerischen Republikanismus. En De Pascale, C., Fuchs, E., Ivaldo, M., Zöller, G., (Eds.). Fichte und die Aufklärung. Hildesheim-Zürich-New York: Georg Olms Verlag.

"Philonenko, A., (2003). Essai sur la philosophie de la guerre. París : Libraire Philosophique Vrin. 
"Villacañas, J. L., (1996). Fichte, el Estado y el derecho internacional. En López Domínguez, V., (Ed.). Fichte. 200 años después (99-116). Madrid: Editorial Complutense.

»Villacañas, J. L., (1999). La nación y la guerra. Confederación y hegemonía como formas de concebir la guerra. Murcia: Diego Marín Librero Editor.

"Von Manz, H. G., (2008). The universality of human rights and the Sovereignity of the State in Fichte's Doctrine of Right. En Rockmore, T., Breazale, D., (Eds.). Rights, Bodies and Recognition. New Essays on Fichte's Foundations of the Natural Right (184-194). Hampshire-Burlington: Ashgate Publishing Company. 
